Revue

Revue de l'histoire des religions

de Ihistoire

des religions

$3 \mid 2012$

Varia

Nicole BÉRIOU - Cécile CABY (éd.), Moines et religieux dans la ville (XII $-\mathrm{XV}^{e}$ siècles)

Toulouse, Privat («Cahiers de Fanjeaux », 44), 2009, 639 p., 1 disque optique numérique avec 163 cartes, plans, dessins et photographies, $18 \mathrm{~cm}, 35 €$, ISBN 978-2-7089-3447-4.

\title{
Valentina Toneatto
}

\section{(2) OpenEdition}

\section{Journals}

Édition électronique

URL : http://journals.openedition.org/rhr/7944

DOI : $10.4000 /$ rhr.7944

ISSN : 2105-2573

Éditeur

Armand Colin

\section{Édition imprimée}

Date de publication : 1 septembre 2012

Pagination : 447-449

ISBN : 978-2200-92975-0

ISSN : 0035-1423

\section{Référence électronique}

Valentina Toneatto, "Nicole bérıou - Cécile caby (éd.), Moines et religieux dans la ville (xIle-xve siècles)》, Revue de l'histoire des religions [En ligne], 3 | 2012, mis en ligne le 04 octobre 2012, consulté le 22 septembre 2020. URL : http://journals.openedition.org/rhr/7944 ; DOI : https://doi.org/10.4000/rhr. 7944

Ce document a été généré automatiquement le 22 septembre 2020

Tous droits réservés 


\section{Nicole BÉRIOU - Cécile CABY (éd.),} Moines et religieux dans la ville (XII ${ }^{e}-\mathrm{XV}^{e}$

\section{siècles)}

Toulouse, Privat («Cahiers de Fanjeaux », 44), 2009, 639 p., 1 disque optique numérique avec 163 cartes, plans, dessins et photographies, $18 \mathrm{~cm}, 35 €$, ISBN 978-2-7089-3447-4.

\section{Valentina Toneatto}

\section{RÉFÉRENCE}

Nicole BÉRIOU - Cécile CABY (éd.), Moines et religieux dans la ville (XII -XVe siècles), Toulouse, Privat ("Cahiers de Fanjeaux », 44), 2009, 639 p., 1 disque optique numérique avec 163 cartes, plans, dessins et photographies, $18 \mathrm{~cm}, 35 €$, ISBN 978-2-7089-3447-4.

1 Le colloque de Fanjeaux de 2008 consacré à l'histoire des Moines et religieux dans la ville a réuni un nombre important de spécialistes des ordres religieux, des chanoines et des moines, ainsi que des spécialistes de l'histoire urbaine du Midi. Les régions du Midi ont connu un développement urbain très marqué à partir du XII siècle et la présence des chanoines, des ordres militaires et mendiants a toujours été très forte, en particulier en Provence et en Languedoc. Jacques Le Goff s'intéresse en premier au lien entre les frères et la ville, en allant jusqu'à «identifier en la présence de couvents mendiants auprès d'une agglomération le meilleur marqueur du fait urbain aux XIII ${ }^{e}-\mathrm{XIV}^{\mathrm{e}} \mathrm{s}$. » (p. 9). Tout en tenant compte des avancées de la recherche concernant les Mendiants ou les chanoines (cf. les Cahiers: Mendiants au Pays d'Oc au XIII siècle, n 8, 1973; Monde des chanoines, $n^{\circ} 24,1989$ ), la rencontre de 2008 s'insère dans cette voie et la renouvelle pour offrir une très bonne livraison des Cahiers de Fanjeaux. Au-delà des études déjà publiées concernant les religieux en milieu urbain, il manquait encore une approche d'ensemble, attentive aux problèmes particuliers de ce champ de recherche. Selon $\mathrm{N}$. Bériou, organisatrice du colloque avec $\mathrm{C}$. Caby, le choix de faire porter la réflexion en 
premier lieu sur la ville, plutôt que sur les ordres religieux qui s'y installent, modifie radicalement l'angle d'attaque qu'avait choisi J. Le Goff. Ce changement de perspective permet de saisir dans toute son ampleur l'importance de la ville - comme espace, lieu d'attraction, cadre de vie - déterminant les rapports avec les institutions urbaines et les citadins ainsi que les pratiques des frères. Le rapport entre la ville et les moines ou les religieux n'est donc plus seulement considéré en des termes d'« évangélisation ", d'«encadrement" (celui-ci étant d'ailleurs particulièrement anachronique) et de conflits, comme cela avait été le cas jusqu'alors. Comme l'indique le titre « Moines et religieux dans la ville » (et non « et la ville»), il s'agit d'un choix historiographique.

Un survol des contenus de ce volume, dense et globalement réussi, permettra de saisir la richesse des contributions et les apports d'une documentation nouvelle ou nouvellement exploitée. On remarquera notamment que l'intérêt porté à l'espace urbain et à celui des établissements religieux, grâce notamment à la place donnée à l'archéologie (F. Guyonnet, N. Pousthomis-Dalle), permet de renouveler les problématiques liées à la présence urbaine des religieux et de revisiter l'approche sociologique et politique des rapports entre la ville et les religieux (D. Riche, $\mathrm{S}$. Balossino et $\mathrm{C}$. Lenoble, N. Coulet). La présence de communautés religieuses urbaines avant le $\mathrm{XII}^{\mathrm{e}}$ siècle est très mal renseignée par manque de sources. Y. Esquieu s'interroge sur ce thème à partir de l'étude de quelques cas mieux connus à Vienne, Arles, Toulouse et Béziers grâce surtout à l'apport des fouilles archéologiques. $\mathrm{J}$. Oberste se penche sur le problème de la concurrence entre les différentes communautés monastiques et canoniales à Toulouse avant l'arrivée des Mendiants, sur l'empreinte de ces mêmes communautés dans la topographie urbaine et sur les relations qu'elles entretiennent avec la société citadine. Les moments clés sont l'arrivée des clunisiens au XI ${ }^{\mathrm{e}}$ siècle et l'implantation des ordres militaires au XII ${ }^{\mathrm{e}}$. Selon $\mathrm{Y}$. Veyrenche, l'abbaye canoniale de Saint-Ruf constitue un terrain d'investigation exemplaire pour évaluer la place des chanoines réguliers dans la société et l'économie urbaines. Les liens entre l'abbaye suburbaine - d'abord située à Avignon puis à Valence dans les années 1150 -, le chapitre cathédral, les évêques et l'obédience de Saint-James montrent un renforcement progressif des relations avec les bourgeois et une accentuation de la participation des chanoines à l'économie urbaine. Le déclin se situe à la fin $d u X^{e}$ siècle en l'absence d'un véritable inurbamento de l'ordre. Les rapports tendus entre l'abbaye de Saint-Pierre de Moissac et la ville (XIII ${ }^{e}-\mathrm{XIV}^{\mathrm{e}} \mathrm{s}$.) sont au centre de la contribution de D. Riche, qui montre les tentatives vaines des habitants pour se libérer de la tutelle monastique clunisienne en ayant recours à l'aide des comtes de Toulouse. Dans ce contexte, les moines noirs ont toujours réussi à bloquer les tentatives d'implantation d'autres ordres, en particuliers des Mendiants. D. Carraz affronte le problème de l'implantation, dès les premières décennies du $\mathrm{XII}^{\mathrm{e}}$ siècle, des commanderies des Templiers et des Hospitaliers en Provence et Languedoc. Soutenus par l'épiscopat et les propriétaires fonciers laïques, les ordres militaires tissent des liens forts avec les acteurs de la vie économique, impriment leur marque sur le paysage urbain et contribuent à l'essor des villes. Pour cela, ils participent d'une transition entre le monachisme traditionnel et la révolution mendiante. La présence cistercienne dans les villes du Midi est l'objet de la contribution d'A. Grélois qui décèle des formes d' inurbamento des moines blancs limitées mais significatives dans le Toulousain et en Gascogne. En prenant le relais, H. Gilles montre que la présence des moines dans les universités, notamment à Toulouse, devient une réalité à partir du pontificat de Benoît XII alors que l'interdiction de quitter le cloître n'est plus respectée dès la fin du 
XIII siècle. L'étude de D. Le Blévec étudie la mise en place du réseau d'établissements religieux à Montpellier à partir du XII ${ }^{e}$ siècle et aux relations qu'ils entretiennent avec la société urbaine et les pouvoirs locaux.

Les deux contributions suivantes ouvrent par l'archéologie la section dédiée au problème de l'établissement des Mendiants en ville, en se penchant sur l'implantation, la topographie et l'architecture des couvents: N. Pousthomis-Dalle propose un important état de la recherche dans le Sud-Ouest de la France et F. Guyonnet offre une excellente synthèse sur les régions du Sud-Est (Comtat Venaissin, Provence, Languedoc oriental). S. Balossino et C. Lenoble, de leur côté, signent un article solide en choisissant de s'attaquer au problème épineux de l'installation des Mineurs à Avignon, que la tradition place en 1227, au moment du siège de la ville par Louis VIII. Mais le seul témoignage de leur présence ces années-là est un acte de vente (1233) d'une maison qui jusqu'alors avait été prêtée aux frères par la commune. Replacée dans le contexte économique, social et politique de la Commune (marqué par un conflit entre la ville et son évêque) et finement analysée, cette transaction, restée jusqu'à présent inconnue, éclaire les liens entre le groupe dirigeant de la ville et les Franciscains, ainsi que le rôle que joue la pauvreté des frères dans la construction de ces liens, en permettant un regard nouveau sur la position politique des Franciscains dans la ville. L'étude remarquable de N. Coulet pour Aix montre l'installation tardive des Mendiants, entre 1245 et 1270 . Grâce à l'étude des testaments (une méthode "désormais classique ", depuis la thèse de J. Chiffoleau, p. 400), on saisit bien les liens entre les Mendiants et les milieux urbains des artisans, des marchands, des notaires et des nobles, mais les formes d'action spirituelle restent plus difficiles à saisir à cause du mauvais état de conservation de la prédication.

4 La relecture des Vitae fratrum de Géraud de Frachet et du De fundatione et prioribus de Bernard Gui par C. Caby montre la construction de la mémoire de la fondation des loca fratrum et la représentation des espaces de vie chez les Prêcheurs. Cet ordre est aussi au centre de la contribution d'A. Dubreil-Arcin qui analyse un dossier hagiographique et liturgique témoignant des tensions entre les cultes des saints locaux et le culte des saints « universels » promu par la réforme liturgique du milieu du XIII ${ }^{e}$ siècle. V. Bouat étudie le rôle des Mendiants dans la conservation et la transmission de la mémoire des ruthénois par la place qui leur est faite dans l'espace conventuel. En reprenant l'analyse de la crise spirituelle de 1316, L. Burnham montre les liens qui unissent les habitants de Narbonne et Béziers aux frères et le soutien politique offert à ces derniers par les consuls. S. Duval s'intéresse aux rapports ambigus entre les monastères féminins et la ville à travers le cas des moniales dominicaines d'Aix et de Montpellier (XIII ${ }^{\mathrm{e}} \mathrm{XIV}{ }^{\mathrm{e}} \mathrm{s}$.). G. Butaud et V. Challet étudient le phénomène de repli urbain des maisons religieuses lié à la guerre dans la province de Narbonne, en Comtat Venaissin et dans le pays d'Apt (milieu XIV ${ }^{\mathrm{e}}$-milieu $\mathrm{XV}$ e siècle), en analysant l'impact des opérations de pillage et de destruction des monastères.

5 Les conclusions d'André Vauchez mettent l'accent sur les acquis du colloque en rupture avec des positions un peu simplistes sur les rapports entre les religieux et la ville, fixant sur l'arrivée des Mendiants le moment de changement et d'innovation, et avec des habitudes historiographiques qui privilégiaient des approches monographiques. 


\section{AUTEURS}

\section{VALENTINA TONEATTO}

Université de Rennes. 